This document is the accepted manuscript version of the following article:

Brouwer, L., Arbelaez, D., Auchmann, B., Bortot, L., \& Stubberud, E. (2019). User defined elements in ANSYS for 2D multiphysics modeling of superconducting magnets. Superconductor Science and Technology, 32(9), 095011 (12 pp.).

https://doi .org/10.1088/1361-6668/ab2e63

\title{
User defined elements in ANSYS for 2D multiphysics modeling of superconducting magnets
}

\author{
Lucas Brouwer ${ }^{1}$, Diego Arbelaez ${ }^{1}$, Bernhard Auchmann ${ }^{2,3}$, \\ Lorenzo Bortot ${ }^{3}$, and Edvard Stubberud ${ }^{3}$ \\ ${ }^{1}$ Lawrence Berkeley National Laboratory, Berkeley, CA, 94720 USA \\ ${ }^{2}$ Paul Scherrer Institute, CH-5232 Villigen, Switzerland \\ ${ }^{3}$ CERN, CH-1211 Geneva 23, Switzerland \\ E-mail: lnbrouwer@lbl.gov
}

\begin{abstract}
Dynamic simulation of superconducting magnets is critical for the design of quench protection systems to prevent potentially damaging temperatures and high voltage from developing after magnet quench. Modeling these scenarios is challenging due to the many multiscale phenomena which impact magnet behavior. These range from conductor scale effects of quench and interfilament coupling currents up to the behavior of the magnet in its powering and protection circuit. In addition, a strong coupling between electromagnetic and thermal domains is required to capture temperature and field dependent material properties and quench behavior. We present a finite element approach which integrates the various effects into the commercial software ANSYS by means of programming new element types. This is shown capable of simulating the strongly coupled transient electromagnetic, thermal, and circuit behavior of superconducting magnets required for quench protection studies. A benchmarking study is presented which shows close agreement between the new ANSYS elements and a COMSOL Multiphysics implementation developed at CERN for dump resistor and Coupling Loss Induced Quench (CLIQ) based magnet protection of a $\mathrm{Nb}_{3} \mathrm{Sn}$ block dipole. Following this, the ANSYS implementation is shown reproducing strongly coupled quench back behavior observed during the test of a $\mathrm{Nb}_{3} \mathrm{Sn}$ superconducting undulator prototype at Lawrence Berkeley National Laboratory.
\end{abstract}

Submitted to: Supercond. Sci. Technol.

Keywords: superconducting magnets, multiphysics modeling, finite element, quench protection, superconducting undulators

\section{Introduction}

Transient behavior of superconducting magnets is frequently determined by multiscale and multiphysics phenomena. A common example of this occurs when a quench protection system (QPS) is activated in an attempt to safely bring down the magnet 
current. To design a system which prevents damage to the magnet, it is critical to be able to accurately simulate the temperature and voltage rise during the current decay. This requires modeling phenomena such as quench back due to eddy currents in the conductor or structural material, current sharing and quench propagation within the conductor, and inductive and resistive coupling of the various effects to the magnet's QPS circuit.

Previous work has focused on simulating these challenging multiphysics problems using laboratory developed finite element codes $[1,2,3,4]$, lumped circuit element models [5], customization of the commercial software COMSOL Multiphysics [6], and the coupling of multiple codes or softwares using co-simulation [7, 8]. We present a new approach using only the commercial finite element software ANSYS [9]. It has previously been demonstrated that ANSYS has the capability to simulate some aspects of the complete problem, such as quench propagation $[10,11,12]$ and the effect of eddy currents in mechanical structures on the current decay of a circuit coupled magnet [13]. We show user defined elements replicating these features and adding the additional missing capabilities of: (1) modeling magnetization of the conductor due to coupling currents and (2) combining all the effects into a single, coupled simulation with field and temperature dependent material properties.

The ability for a user to define their own element type is a documented feature of ANSYS, for which the authors are aware of two previous examples relevant to electromagnetic applications $[14,15]$. The creation of a user element is accomplished by writing the Fortran code which defines the element's properties and builds the finite element matrices. A custom ANSYS executable is then compiled which allows for the use of this element as if it were included in the standard distribution (making it compatible with all geometry generation, meshing, solving, and post-processing features). User programmed generation of the element matrices gives full control over the choice of element shape functions, integration points, material properties, and FEM formulation.

Two elements are used to implement a custom FEM approach, with the first being an electromagnetic element with optional coupling to an external circuit. An equivalent magnetization term is included in the vector potential formulation to model interfilament coupling loss (IFCL) within the conductor. A second, thermal element is used to model the temperature rise due to quench induced ohmic heating and IFCL. These two elements are coupled using the Multi-field Solver in ANSYS such that magnetic field, temperature, and various loads are shared between them (see figure 1). We present an initial benchmarking study with results for a $\mathrm{Nb}_{3} \mathrm{Sn}$ dipole magnet compared between this approach and a similar implementation in COMSOL Multiphysics [16]. Following this, behavior predicted by the user elements is compared to data taken during the test of a prototype $\mathrm{Nb}_{3} \mathrm{Sn}$ undulator magnet at Lawrence Berkeley National Laboratory. 
Electromagnetic Field

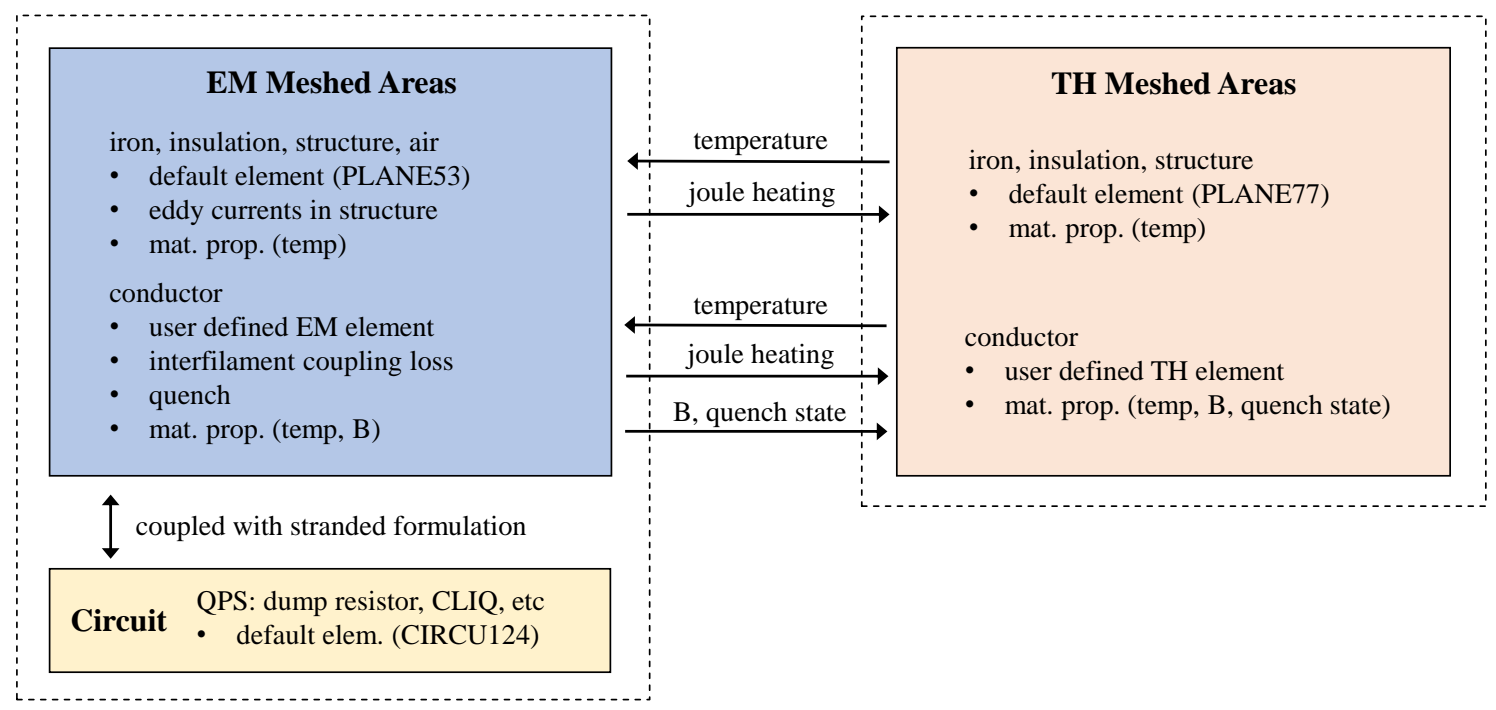

Figure 1. An overview of coupled electromagnetic, circuit, and thermal simulation in ANSYS with user defined elements is shown. Such an approach allows for simulating the impact of interfilament coupling loss, quench, and structural eddy currents on magnet behavior while including temperature and field dependent material properties. The independently meshed electromagnetic and thermal domains are coupled using the Multi-field Solver as described in section 3.

\section{The finite element model}

An electromagnetic and thermal model was developed for use in conductor regions where superconducting effects are desired (as illustrated in figure 1). These models were implemented in ANSYS by the creation of two user elements. This approach integrates the desired effects at the point of element matrix generation, no longer requiring manual updating of superconducting properties between a stop and restart of the solver as implemented in previous work [11, 12]. The thermal model follows directly from ANSYS (see documentation in [17]), with extended capabilities of programmable material property fits and automatic quench checking and heat generation. The material properties are homogenized during element matrix generation based on specified fractions of conductor, superconductor, and insulation using a method similar to what is described in [6]. The electromagnetic model is based on the vector potential approach used in ANSYS, with modifications to the formulation made to include the effects of quench and interfilament coupling loss. The following subsections describe this model.

\subsection{Vector potential with equivalent magnetization for IFCL}

The default approach to modeling eddy currents in ANSYS uses the $\boldsymbol{A}, \mathrm{V}-\boldsymbol{A}$ formulation with both vector potential $\boldsymbol{A}$ and electric scalar potential $\mathrm{V}$ degrees of freedom in conducting regions [18], requiring modeling and meshing of the conductive paths in 
which the induced currents flow. This is impractical for the simulation of interfilament coupling currents in a 2D magnet cross section due to filament sizes on the order of 5 to $100 \mu \mathrm{m}$ and the 3D nature of the induced current path. An alternative approach, which does not require a mesh density on the same order of the induced currents, assumes a predetermined current path producing an equivalent magnetization, with time constant $\tau$, of

$$
\boldsymbol{M}_{\boldsymbol{e}}=-\frac{2 \tau}{\mu_{0}} \frac{\partial \boldsymbol{B}}{\partial t}
$$

This approach is found applied to the case of a twisted filamentary composite in a uniform, changing transverse field (relevant for 2D simulations of multifilamentary superconducting strands) in $[19,20]$ and has been implemented in several magnet modeling codes $[1,5,6]$. With assumptions about the filament layout within the strand and the resulting current loops, an interfilament coupling loss (IFCL) time constant of

$$
\tau=\frac{\mu_{0}}{2 \rho_{e t}}\left(\frac{L}{2 \pi}\right)^{2},
$$

is written in terms of an effective transverse resistivity of the strand matrix $\rho_{e t}$ and filament twist pitch $L$ along the length of the strand. Limitations of this approximation and a more detailed approach can be found summarized in [21]. The induced currents deposit energy as heat within the strand matrix with a power per unit volume of

$$
P_{e}=M_{e} \cdot \frac{\partial \boldsymbol{B}}{\partial t}
$$

which in many cases leads to IFCL being an effective quench back mechanism.

The equivalent magnetization approach includes the effects of eddy currents without the need for an additional degree of freedom (DOF), and the finite element formulation in $3 \mathrm{D}$ is derived from the vector potential only,

$$
\nabla \times \nu \nabla \times \boldsymbol{A}-\nabla \times \boldsymbol{M}_{\boldsymbol{e}}=\boldsymbol{J}_{\boldsymbol{s}}
$$

with $J_{s}$ as a source current density and $\nu=\mu^{-1}$. Considering the form of $\boldsymbol{M}_{\boldsymbol{e}}$, the differential equation to be solved using the FEM is

$$
\nabla \times \nu \nabla \times \boldsymbol{A}+\nabla \times 2 \tau \nu \nabla \times \frac{\partial \boldsymbol{A}}{\partial t}=\boldsymbol{J}_{\boldsymbol{s}}
$$

Here it is seen the addition of the magnetization term introduces a damping matrix of similar form (curl-curl) as the stiffness matrix used for a typical vector potential element. To implement the FEM, the weak integral of equation 5 is taken with test functions chosen to be the same as the shape functions carrying the DOF within the element [18]. This leads to

$$
\left[K^{A A}\right]\{A\}+\left[C^{A A}\right] \frac{\partial}{\partial t}\{A\}=\left\{J^{s}\right\}
$$


from which the element stiffness matrix $\left[K^{A A}\right]$, damping matrix $\left[C^{A A}\right]$, and load vector $\left\{J^{s}\right\}$ are extracted. With the vector potential within the element written in terms of the shape function matrix $\left[N_{A}\right]$ and the DOF values at the element nodes $\left\{A_{e}\right\}$,

$$
\{A\}=\left[N_{A}\right]^{T}\left\{A_{e}\right\},
$$

the resulting element matrices and load vector are

$$
\begin{gathered}
{\left[K^{A A}\right]=\frac{1}{\mu_{0}} \int\left[\left(\nabla \times\left[N_{A}\right]^{T}\right)^{T}\left(\nabla \times\left[N_{A}\right]^{T}\right)\right] d V_{\text {elem }}} \\
{\left[C^{A A}\right]=\frac{2 \tau}{\mu_{0}} \int\left[\left(\nabla \times\left[N_{A}\right]^{T}\right)^{T}\left(\nabla \times\left[N_{A}\right]^{T}\right)\right] d V_{\text {elem }}} \\
\left\{J^{S}\right\}=\int\left\{J_{S}\right\}\left[N_{A}\right]^{T} d V_{\text {elem }} .
\end{gathered}
$$

Here the equations are given in general 3D form in terms of the nodal potential (assuming $\left.\{A\}=\left\{A_{x}, A_{y}, A_{z}\right\}\right)$. For the $2 \mathrm{D}$ user element these simply reduce to a single component $A_{z}$, and are evaluated using Gaussian quadrature with similar shape functions and integration points as used by ANSYS.

\subsection{Coupling to an external circuit as a stranded conductor}

Regions modeled as coils are coupled to an external circuit following the method developed by ANSYS [22], with several modifications to account for IFCL, quench effects, and separate effective lengths for coil resistance and inductance. A stranded formulation is used which adds a current $i$ and voltage $e$ DOF to the vector potential given in equation 5. Both these new DOF are constrained to be single values for a modeled coil region, with $i$ being the current per stranded coil turn, and $e$ being the voltage drop across the coil. The voltage drop is made up of both a resistive $e_{R}$ and inductive $e_{L}$ contribution such that

$$
e=e_{R}+e_{L}
$$

For a general stranded coil of fixed cross section and resistivity $\rho$, the resistive voltage is given by

$$
e_{R}=i\left(\frac{N_{c}}{S_{c}}\right)^{2} \int \rho d V,
$$

where $N_{c}$ is the number of coil turns and $S_{c}$ is the modeled coil area. As will be described in section 2.3, quench and current sharing effects are accounted for using a single parameter $I_{f c u}$ representing the fraction of current assumed to be flowing in the stabilizing material compared with the superconductor. With this assumption, the contribution to the resistive voltage is determined by the fractional area of stabilizer 
and the amount of current assumed to be flowing in this region. Adjusting equation 12 to account for these assumptions, the resistive voltage drop is given by

$$
e_{R}=i L_{c}\left(\frac{N_{c}}{S_{c}}\right)^{2} \frac{I_{f c u}}{f_{c o n d}\left(1-f_{s c}\right)} \int \rho_{s t} d S,
$$

where $L_{c}$ is introduced as an effective length chosen to best match the resistance of the $3 \mathrm{D}$ coil, $f_{\text {cond }}$ defines the fraction of the total coil area which is conductor, $f_{s c}$ defines the fraction of superconductor within this conductor area, and $\rho_{s t}$ is the resistivity of the stabilizing material. In case of multi-strand cables the factor $L_{c}$ is also accounting for the transposition pitch.

The inductive voltage is given by the time derivative of the linked flux

$$
e_{L}=\frac{\partial \Phi}{\partial t}=N_{c} \frac{\partial}{\partial t} \int(\hat{t} \cdot \boldsymbol{A}) d S
$$

which for a $2 \mathrm{D}$ model with current restricted to flow only in the $z$ direction is given by

$$
e_{L}=L_{i} \frac{N_{c}}{S_{c}} t \int \frac{\partial A_{z}}{\partial t} d S
$$

Here the variable $t$ is positive or negative one based on the direction of the current, and $L_{i}$ is introduced as an effective length scaling of the inductance chosen to match the 3D magnet.

Circuit coupling is accomplished by the addition of equation 11 to the original vector potential formulation in equation 6 (with the source current density now derived from the current per turn DOF using the proper winding function relations in the $K^{A i}$ matrix). This leads to the coupled equations

$$
\begin{gathered}
{\left[C^{A A}\right] \frac{\partial}{\partial t}\{A\}+\left[K^{A A}\right]\{A\}+\left[K^{A i}\right]\{i\}=\{0\}} \\
{\left[C^{e A}\right] \frac{\partial}{\partial t}\{A\}+\left[K^{e e}\right]\{e\}+\left[K^{e i}\right]\{i\}=\{0\},}
\end{gathered}
$$

where the matrices shown are matched to the DOF dependence of each term. With respect to the original ANSYS model, a new matrix $C^{A A}$ is included for interfilament coupling loss, and the voltage balance matrices $K^{e i}$ and $C^{e A}$ are derived from equations 13 and 15 to include the changes mentioned. The discrete form for the circuit coupled element (similar to equations 8-10), and the method by which the $e$ and $i$ DOF's are coupled to other external circuit elements can be found in the appendix.

\subsection{Current sharing and quench}

The stranded formulation assumes a uniform current density and therefore does not solve for the distribution of current within the superconductor or stabilizing regions which changes based on the superconducting properties and quench behavior (this could be done, for example, with $\boldsymbol{A}-V$ or $\boldsymbol{H}$ formulations). The choice of a stranded approach 
is motivated by modeling of multifilamentary conductors typical of $\mathrm{Nb}-\mathrm{Ti}$ and $\mathrm{Nb}_{3} \mathrm{Sn}$ magnets. In order to capture the impact of the effect of quench on ohmic loss and resistance growth, a single scaling parameter $I_{f c u}$ is used to represent the fraction of current assumed to be in the stabilizing material. With this definition, three distinct quench states can be defined as shown in table 1.

Table 1. Quench States

\begin{tabular}{lc}
\hline Regime & Fraction of Current in Stabilizer \\
\hline Fully Superconducting & $I_{f c u}=0$ \\
Current Sharing & $0<I_{f c u}<1$ \\
Fully Quenched & $I_{f c u}=1$ \\
\hline
\end{tabular}

The conductor is assumed to have a critical surface parametrized by magnetic field, temperature, and transport current density beneath which it is fully superconducting. Strain dependence of this critical surface is neglected at this time. Above the critical surface, some (current sharing) or all (fully quenched) of the current is assumed to move from the superconductor into the stabilizing material, leading to resistive loss. Accurate assumptions for the variation of $I_{f c u}$ during current sharing is dependent on the superconducting material and the significance of this quench state to magnet behavior being simulated. An exhaustive review of this topic is found in Chapter 18 of [23]. A common assumption for the current sharing regime of $\mathrm{Nb}_{3} \mathrm{Sn}$ and $\mathrm{Nb}-\mathrm{Ti}$ conductor is a linear variation of $I_{f c u}$ with temperature $T$

$$
I_{f c u}=1-\frac{T_{c B}-T}{T_{c B}-T_{c s}}
$$

from the point at which the critical current equals the transport current $T_{c s}$ up to the temperature $T_{c B}$ at which the transport current is equal to zero in the superconductor $[24]$.

\subsection{Homogenized joule heating}

The two sources of element heating are resistive loss due to quench and IFCL. These losses are homogenized to account for fill factors of conductor and superconductor within the modeled coil region. The parameter $f_{\text {cond }}$ is used to define the fraction of the total coil area which is conductor, and $f_{s c}$ to define the fraction of superconductor within this conductor area. Quench induced loss is assumed to occur only within the stabilizer of the conductor, with the magnitude dependent on the quench state (see table 1) and the resistivity of the stabilizing material. If $J_{e}$ is the element current density, the power per unit volume of modeled conductor is then given by

$$
P_{\text {res }}=\rho_{s t} \frac{\left(I_{f c u} J_{e}\right)^{2}}{f_{\text {cond }}\left(1-f_{s c}\right)}
$$


for quench based loss, and

$$
P_{e}=2 \tau \frac{f_{\text {cond }}}{\mu_{0}}\left|\frac{d B}{d t}\right|^{2}
$$

for losses resulting from interfilament coupling currents (see equation 3). For a given quench state, the temperature and field dependence of these losses is driven by the variation of the stabilizer's resistivity $\rho_{s t}$. For $\mathrm{Nb}-\mathrm{Ti}$ and $\mathrm{Nb}_{3} \mathrm{Sn}$ this stabilizer is typically a high RRR copper, resulting in $\rho_{s t}$ (and then also $\tau$ for which $\rho_{\text {et }}$ in equation 2 also depends on $\rho_{s t}$ ) showing strong temperature dependence and magnetoresistive effects.

\section{Coupled simulation using the Multi-field Solver}

The ANSYS Multi-field Solver allows for solving of sequentially coupled problems with independent meshes. A unique, meshed region is generated for each physics field and load coupling interfaces for which loads will be passed between them are specified. Each region is solved independently with its own time stepping and solution options. The solver transfers the loads across the defined interfaces (even with dissimilar meshes), and iterates between each physics field in sequence until the transfer of loads converges for a user defined "stagger" time step as shown in figure 2.

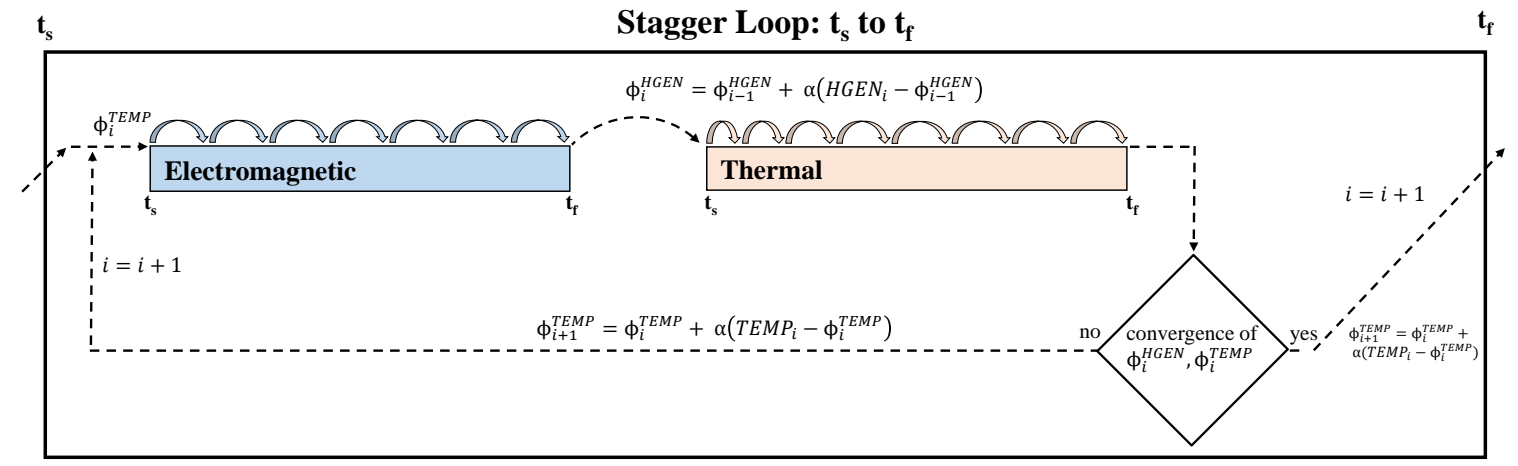

Figure 2. A stagger loop within the Multi-field Solver is shown for coupled electromagnetic and thermal fields (see figure 1 for an example of how such a simulation is set up with the user elements). In this example, the loads transferred between fields are heat generation $\phi^{H G E N}$ and temperature $\phi^{T E M P}$. This approach loops over the stagger time step (from $t_{s}$ to $t_{f}$ ) with a relaxation factor $\alpha$ applied to the load transfer until convergence of the loads is achieved. Separation of the problem into sequentially defined stagger steps is used to simulate over the entire time domain.

This solver has been successfully used for fully coupled simulations including the user elements (for example see the verification study in section 4). To do this, two physics fields are created which are shown labeled as "electromagnetic" and "thermal" in figure 1. A load transfer interface is specified between meshed coil regions and any structural regions with eddy currents. This allows for passing Joule heat loads from 
the electromagnetic region to the thermal region, and passing temperature back. Both temperature and Joule heating are standard loads which may be transferred with the Multi-field Solver. To allow for thermal material properties to also vary with magnetic field and quench state, a workaround using a shared module was implemented to pass these two variables in a non-standard use of the solver.

\section{Benchmarking with COMSOL}

A verification study was completed comparing results from the ANSYS user elements to a similar 2D FEM implementation in COMSOL developed at CERN [6] within the STEAM project [25]. The results from the full study are found in [26]. Effects such as quench resistance, yoke saturation, IFCL, and structural eddy currents were compared across several models with good agreement found. We present the results of one such study which focuses on a simplified $\mathrm{Nb}_{3} \mathrm{Sn}$ dipole magnet protected with a dump resistor and Coupling Loss Induced Quench (CLIQ) [27].

\subsection{The $\mathrm{Nb}_{3} \mathrm{Sn}$ dipole model}

A dipole model was designed to allow for comparison of results in a regime representative of realistic $\mathrm{Nb}_{3} \mathrm{Sn}$ accelerator magnets. This model is shown in figure 3, and a list of high level parameters are given in table 2 .

Table 2. Overview of the $\mathrm{Nb}_{3} \mathrm{Sn}$ Dipole Model

\begin{tabular}{lcc}
\hline Parameter & Value & Unit \\
\hline inner layer turns (per quadrant) & 14 & \\
outer layer turns (per quadrant) & 18 & \\
turn width & 1.5 & $\mathrm{~mm}$ \\
turn height & 15 & $\mathrm{~mm}$ \\
strands (per turn) & 40 & \\
strand diameter & 0.75 & $\mathrm{~mm}$ \\
filament twist pitch & 14 & $\mathrm{~mm}$ \\
$f_{\text {eff }}$ with $\rho_{\text {et }}=f_{\text {eff }} \rho_{\text {st }}$ & 1.0 & \\
Cu RRR & 200 & \\
non-Cu fraction & 0.4 & \\
Nb ${ }_{3}$ Sn $\mathrm{J}_{c}$ (4.5 K, $\left.12 \mathrm{~T}\right)$ & 2040 & $\mathrm{~A} / \mathrm{mm}{ }^{2}$ \\
short-sample current $(4.5 \mathrm{~K})$ & 15.37 & $\mathrm{kA}$ \\
short-sample cond. field $(4.5 \mathrm{~K})$ & 11.7 & $\mathrm{~T}$ \\
Lc: effective res. coil length & 10.11 & $\mathrm{~m}$ \\
Li: effective ind. coil length & 9.2 & $\mathrm{~m}$ \\
\hline
\end{tabular}


(a)
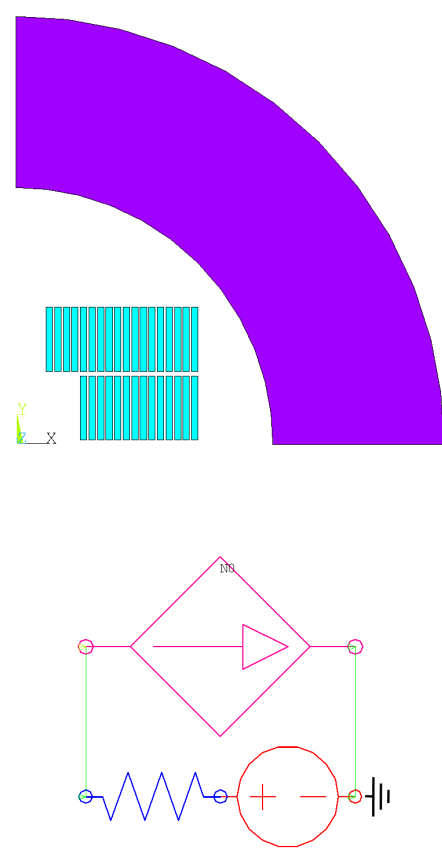

(b)
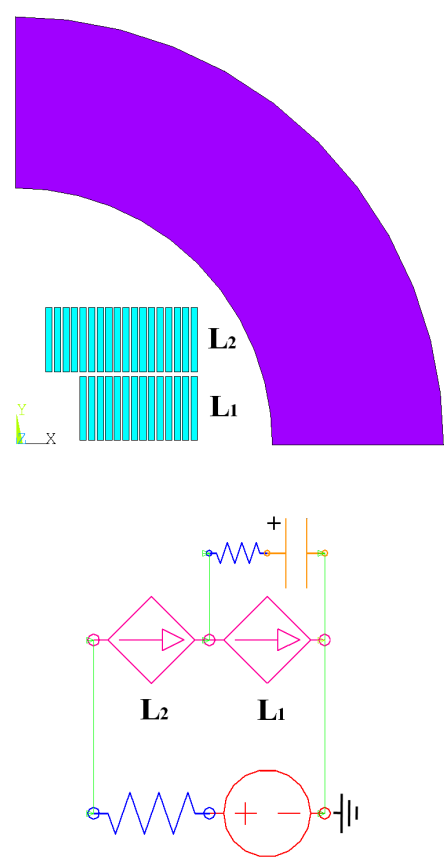

Figure 3. The two conductor layers and iron yoke of the $\mathrm{Nb}_{3} \mathrm{Sn}$ dipole ANSYS model are shown with the dump resistor circuit (a) and the CLIQ circuit (b). The DOF of the meshed conductor regions of these models are coupled to their respective circuits, allowing for consistent simulation of both electromagnetic and circuit effects. A similar model consisting of only the conductor region is used for the thermal domain during the Multi-field solution

\subsection{Protection with a dump resistor}

A first comparison between ANSYS and COMSOL was performed for a dump resistor extraction exhibiting strongly coupled electromagnetic and thermal behavior. A simple circuit was built using CIRCU124 elements consisting of a resistor, voltage source, and a stranded coil element coupled in $e$ and $i$ to the coil region in the meshed model (see figure 3). A dump resistor value of $30 \mathrm{~m} \Omega$ was used for all tests. The simulation begins with the magnet operating in a static condition at $4.5 \mathrm{~K}$ and $13.8 \mathrm{kA}$. This operating point is slightly less than $90 \%$ of the magnet's short-sample limit. At $5.0 \mathrm{~ms}$ the voltage source is ramped down to zero over $0.1 \mathrm{~ms}$ to effectively put the magnet in series with the dump resistor only. The full details of this simulation including the material property fits are found in [26].

The behavior of the magnet was studied with increasing levels of detail as outlined in table 3. A first simulation was performed with no IFCL or quench losses, making the current decay dependent only on the magnet's inductance. A second simulation added IFCL which influences the current decay by changing the differential inductance of the magnet. For this case, coupling to a thermal model was also included to capture effects on $\tau$ due to changes in material properties from heating of the conductor. For the final simulation, current sharing and quench were added to the previous case, allowing for 
IFCL induced quench resistance growth.

Table 3. Dump Resistor Verification Tests

\begin{tabular}{lcccc}
\hline & Yoke Saturation & IFCL & Thermal Coupling & Quench \\
\hline No Loss & $\mathrm{x}$ & & & \\
IFCL Only & $\mathrm{x}$ & $\mathrm{x}$ & $\mathrm{x}$ & \\
IFCL w/Quench & $\mathrm{x}$ & $\mathrm{x}$ & $\mathrm{x}$ & $\mathrm{x}$ \\
\hline
\end{tabular}

Agreement between ANSYS and COMSOL results for the magnet current decay is shown in figure 4 for the final simulation case outlined in table 3 . This magnet exhibits strong quench back behavior with IFCL heating the coil to quench, after which the coil resistance growth rapidly increases rate of magnet current decay. This is further illustrated by figure 5 where the rapid growth of hotspot temperature and coil resistance in the final, fully coupled case (IFCL w/Quench) is compared between codes. The energy loss for each mechanism is compared to the total change in energy of the system in table 4 .

Table 4. Energy Loss Comparison for IFCL w/Quench Case

\begin{tabular}{lllll}
\hline Location & \multicolumn{2}{c}{ ANSYS } & \multicolumn{2}{c}{ COMSOL } \\
\hline & E (kJ) & \multicolumn{1}{c}{$\%$} & E (kJ) & $\%$ \\
Dump Res. & 505.96 & 34.45 & 497.25 & 33.80 \\
IFCL & 14.32 & 0.98 & 14.24 & 0.97 \\
Coil Res. & 948.24 & 64.56 & 959.55 & 65.23 \\
\hline
\end{tabular}

$\%$ energy deposition is based on a total energy change between 5 and $500 \mathrm{~ms}$.

\subsection{Protection with $C L I Q$}

A second study compares results for a Coupling Loss Induced Quench (CLIQ) discharge [27] in a layer-layer configuration as shown in figure 3. CLIQ is a protection scheme which seeks to quench large portions of the magnet coil in a distributed fashion in order to rapidly bring down the magnet current using coil quench resistance. At the detection of a quench, a capacitor bank is discharged across one or more sections of the magnet coil which generates an oscillation of current in the coil sections about the nominal magnet transport current (see figure 6). The resulting field oscillations induce interfilament coupling currents which heat portions of the coil to quench. This approach is typically considered for large inductance accelerator magnets where dump resistor based protection is no longer possible due to voltage and hotspot temperature constraints. CLIQ protection scales well to large inductance magnets because the current 


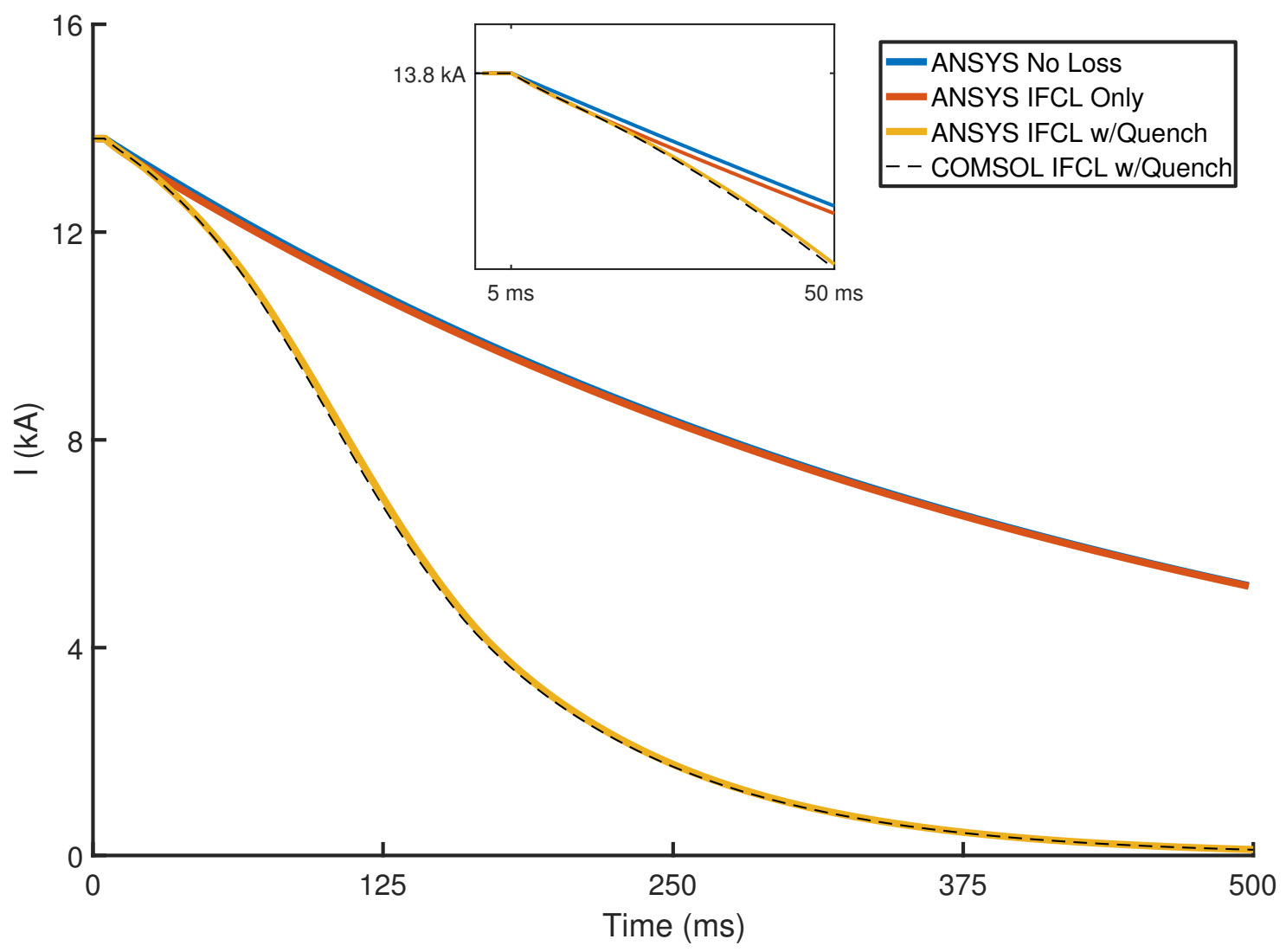

Figure 4. ANSYS and COMSOL results are compared for a dump resistor extraction exhibiting strongly coupled electromagnetic and thermal behavior. Effects are built up from no losses, to only IFCL, and finally to a fully coupled case where quench and IFCL are considered. A slight difference in the initial current decay between the "No Loss" and other two cases is seen up to about $20 \mathrm{~ms}$. Here the coil is not yet quenched, but the influence of coupling currents on the magnet's differential inductance causes the current to decay faster. At $20 \mathrm{~ms}$, quench resistance growth begins to play a dominant role as "IFCL w/Quench" quickly decays away from "IFCL Only". This is an example of quench back, where the rapid field change initiated by the dump resistor induces IFCL which heats the coil to quench. The resistance rise due to quench drives the current down much faster than a case where the effects of IFCL and quench are not considered.

oscillations are induced between sections of the magnet, and the relevant inductance for inducing these currents is much lower than total magnet inductance.

In this comparison, the dump resistor in the main circuit is set to zero, and a CLIQ resistor of $15 \mathrm{~m} \Omega$ and capacitor of $35 \mathrm{mF}$ are added. For the results shown, the capacitor was charged to $350 \mathrm{~V}$. The CLIQ system is activated between 5.00 and 5.01 ms with the magnet previously set up in a static condition at $4.5 \mathrm{~K}$ and $13.8 \mathrm{kA}(90 \%$ of short-sample). The CLIQ discharge induces current oscillation between the magnet layers on a similar scale of the IFCL time constant (see the no loss case in figure 6). Figures 7 and 8 show how the IFCL heating induced by this oscillation drives the coil to quench, with the resulting coil resistance growth bringing down the magnet current. Comparison between the ANSYS and COMSOL results in these figures show excellent 


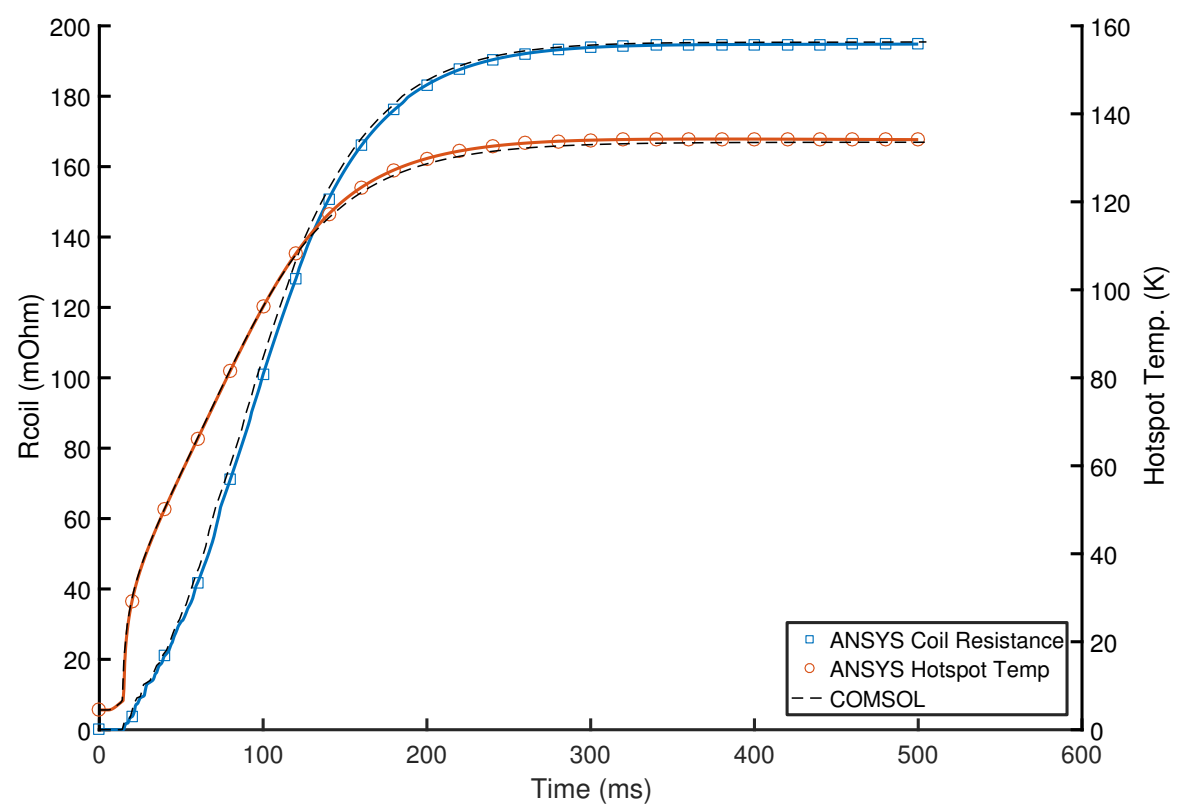

Figure 5. The rise in coil resistance and peak coil temperature is compared between ANSYS and COMSOL for the final, fully coupled simulation (IFCL w/Quench).

agreement for this fully coupled electromagnetic, thermal, and circuit simulation.

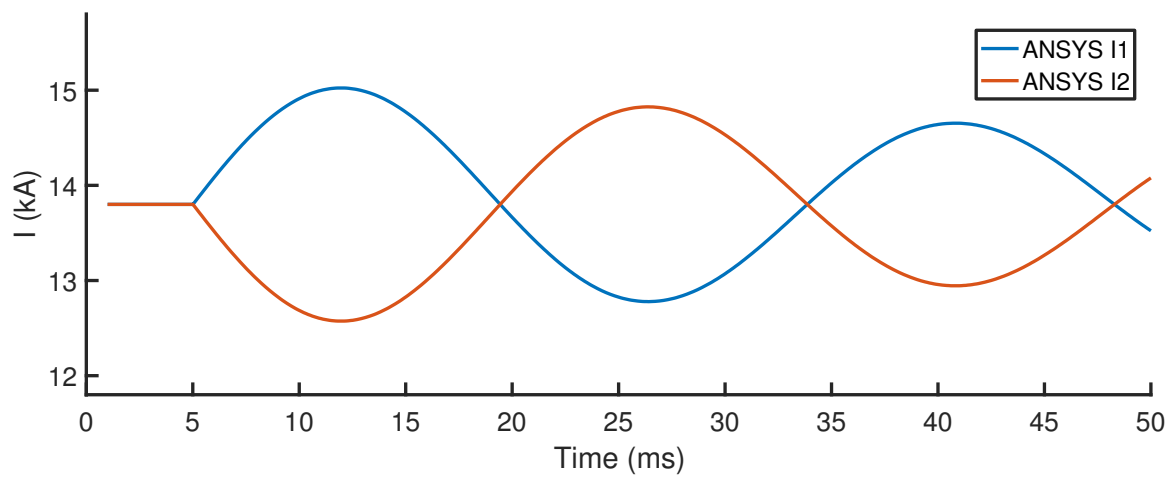

Figure 6. The induced current oscillation in the two coil sections about the starting current of $13.8 \mathrm{kA}$ from a CLIQ discharge is shown for a no loss case. The oscillation period is similar to the IFCL time constant in the high field region. 


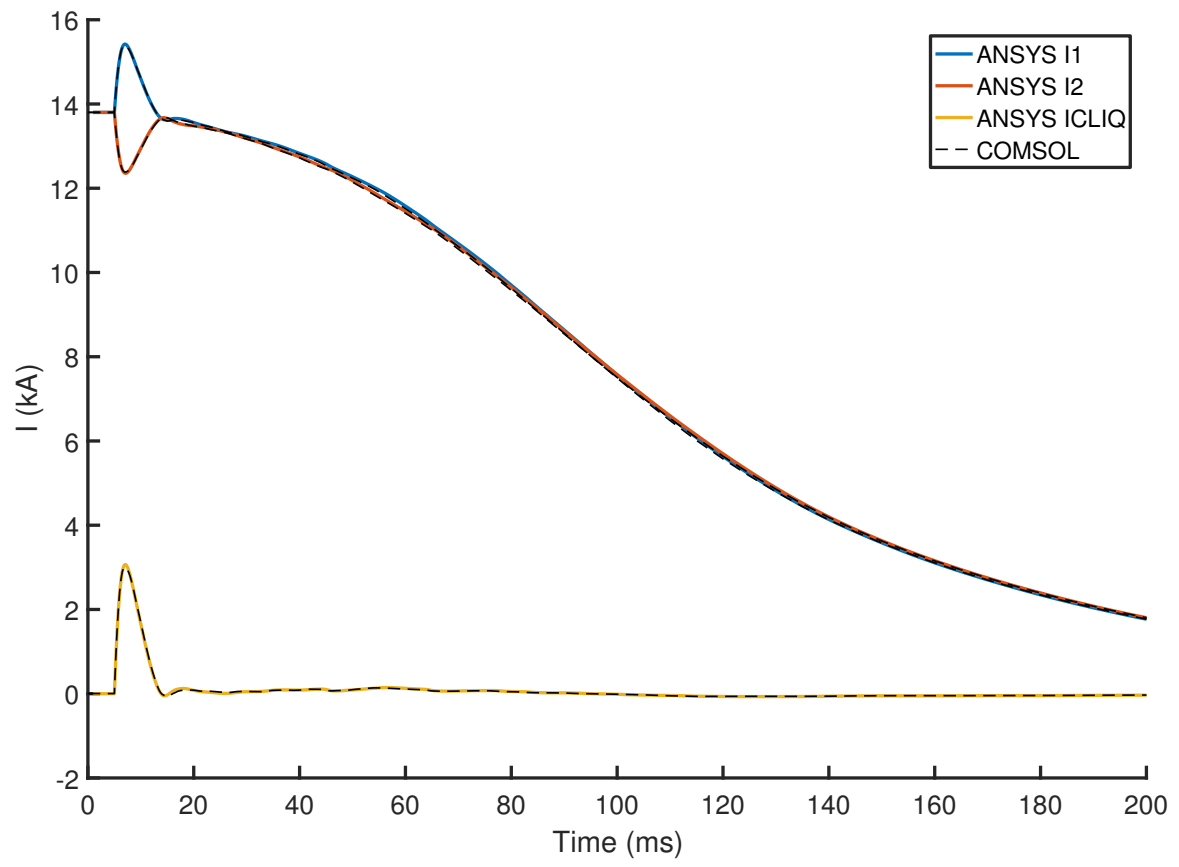

Figure 7. The currents in the different coil sections following a CLIQ discharge are compared between the ANSYS and COMSOL models. The induced field oscillations generate IFCL heating which leads to quenching of a large portion of the coil (also see figure 8).

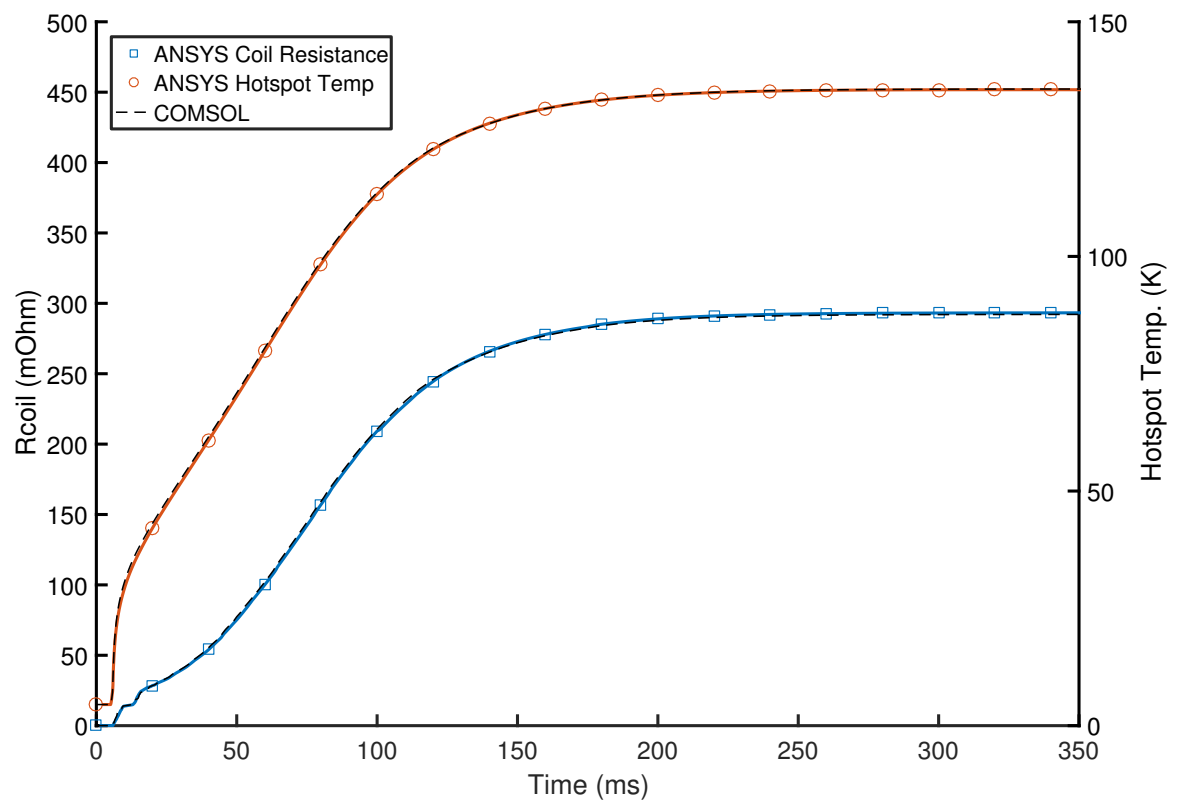

Figure 8. The rise in coil resistance and peak coil temperature is compared between ANSYS and COMSOL following the CLIQ discharge. 


\section{Comparison to $\mathrm{Nb}_{3} \mathrm{Sn}$ undulator test data}

A series of $\mathrm{Nb}_{3} \mathrm{Sn}$ superconducting undulators (SCUs) were built and tested at Lawrence Berkeley National Laboratory as part of a R\&D program for LCLS-II and future free electron lasers [28]. These magnets consist of two ferromagnetic iron cores with machined pockets along the length. The pockets are wound with superconducting strand such that the current polarity changes from one pocket to the next along the length. The two cores are assembled together with a gap left between them for an electron beam. The interaction of the beam with the alternating fields generated by the SCU serves as the radiation source in free electron lasers.

These magnets are well suited for benchmarking the user elements due to: (1) the use of single strands eliminating cable based coupling currents found in many other $\mathrm{Nb}_{3} \mathrm{Sn}$ magnets and (2) exhibiting strong quench back behavior changing as a function of field level. The test of a short prototype SCU was selected for a first comparison due to existing data for a series of pre-quench, dump resistor extractions at increasing levels of initial magnet current. This allows for benchmarking of the user elements with test data over a wide range quench back behavior. An overview of the properties of this magnet is found in table 5 and a picture of the cross section can be seen in figure 9 . This prototype corresponds to a short, $80 \mathrm{~cm}$, length single core, whereas the final SCU magnet includes two cores of $1.4 \mathrm{~m}$ length assembled together.

Table 5. Overview of the $\mathrm{Nb}_{3} \mathrm{Sn}$ SCU Short Model

\begin{tabular}{lcc}
\hline Parameter & Value & Unit \\
\hline period length & 19 & $\mathrm{~mm}$ \\
pocket width & 6.32 & $\mathrm{~mm}$ \\
pocket height & 4.67 & $\mathrm{~mm}$ \\
powered turns per pocket (left to right in sym.) & $7-35-56-56$ & \\
strand architecture & $132 / 169 \mathrm{RRP}{ }^{\circledR}$ & \\
strand diameter & 0.6 & $\mathrm{~mm}$ \\
filament twist pitch & 12 & $\mathrm{~mm}$ \\
$\mathrm{Cu} \mathrm{RRR}$ & 250 & \\
non-Cu fraction & 0.45 & \\
$f_{\text {eff }}$ with $\rho_{\text {et }}=f_{\text {eff }} \rho_{s t}$ & 4.0 & \\
effective resistive coil length $\left(L_{c}\right)$ & 97.1 & $\mathrm{~mm}$ \\
effective inductive coil length $\left(L_{i}\right)$ & 90 & $\mathrm{~mm}$ \\
Nb ${ }_{3} \mathrm{Sn} \mathrm{J}_{c}(4.5 \mathrm{~K}, 10 \mathrm{~T})$ & 2880 & $\mathrm{~A} / \mathrm{mm}^{2}$ \\
short-sample current $(4.5 \mathrm{~K})$ & 965 & $\mathrm{~A}$ \\
short-sample cond. field $(4.5 \mathrm{~K})$ & 5.2 & $\mathrm{~T}$ \\
\hline
\end{tabular}

The ANSYS model was matched to the test configuration of a single, short prototype magnet as seen in figure 9 . The effective lengths $L_{c}$ and $L_{i}$, which scale resistive and inductive effects from $2 \mathrm{D}$ to $3 \mathrm{D}$, were chosen to match the physical length of the coil and the linked flux of a 3D ANSYS model. The use of a large scaling factor 

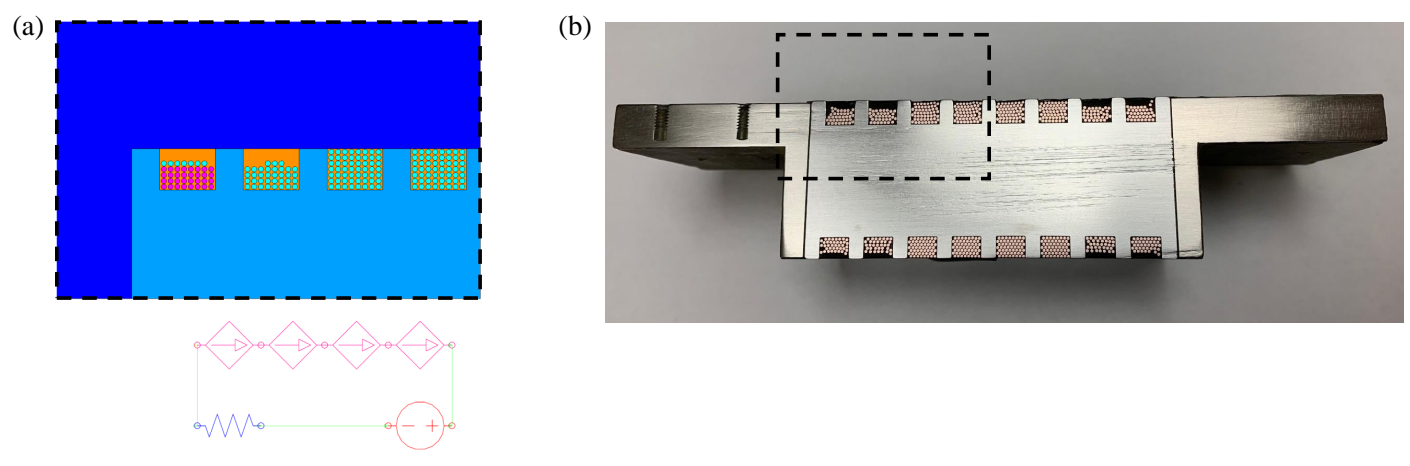

Figure 9. (a) The electromagnetic ANSYS model is shown in a dump resistor circuit. The meshed regions consist of ferromagnetic low carbon steel (light blue), un-powered corrector turns (magenta), powered turns (teal), glass filled epoxy (orange), and air which extends beyond the top and left boarders for far field boundary conditions (dark blue). This is coupled to a similar thermal region (without air) using the Multi-field Solver to simulate IFCL induced quench back in an external dump resistor protection circuit. (b) A cross-section of the short prototype $\mathrm{Nb}_{3} \mathrm{Sn}$ undulator with the symmetric region marked.

$f_{\text {eff }}$ between the matrix resistivity and an effective resistivity for all coupling losses is based on measurements made on a similar $\mathrm{Nb}_{3} \mathrm{Sn}$ strand [29, 30]. For each case, a single static solution was first solved with the voltage source set to produce the initial current matching the test. This source was then ramped to zero over $0.1 \mathrm{~ms}$ to effectively place the magnet in series with a fixed resistance dump resistor for current extraction. The solution proceeds with transient effects using the Multi-field Solver for coupling of independently meshed electromagnetic and thermal domains as described in section 3 .

To match data taken during the test, current extractions with no initial quench were simulated from 400 to 800 A using a dump resistor of $48.1 \mathrm{~m} \Omega$. Figure 10 shows the current decay curves from these simulations including cases with and without IFCL and quench effects. When normalized to peak current, the "No Loss" cases show only a small variation with initial current which is the result of non-linear magnetization of the iron core with field level. At the 400 A level, the "IFCL w/Quench" case remains superconducting due to IFCL heating not being able to overcome the large margin to quench. This results in a current decay which shows little deviation from the no loss case. As the initial current increases up to $800 \mathrm{~A}$, the margin is reduced and IFCL heating grows. At a certain level this begins to induce quench, adding resistance to the circuit and driving the current down faster (quench back). As expected, the degree of quench back is seen increasing with initial current (this is particularly clear when comparing the normalized current decay).

A sensitivity study of the ANSYS results to input parameters and material property fits was performed to allow for a more detailed comparison to test data. Table 6 summarizes this study and the results. The deviation from the nominal case was evaluated on a parameter by parameter basis by comparing both the quench integral and 
a)

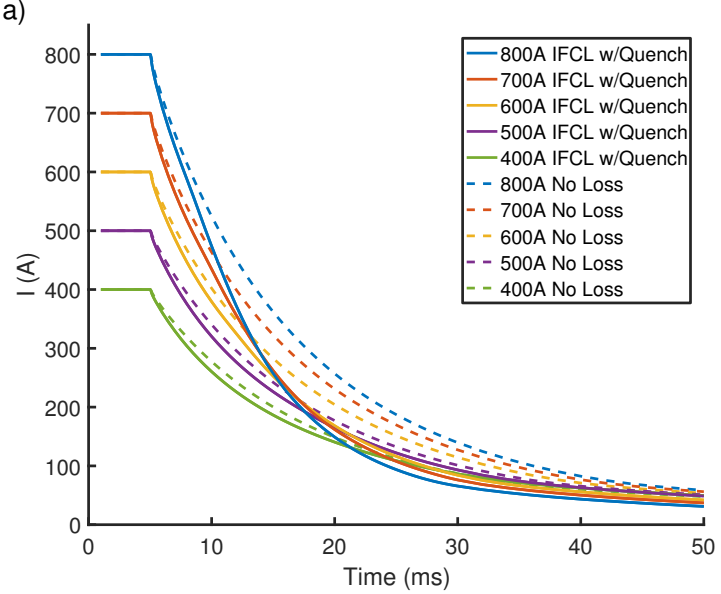

b)

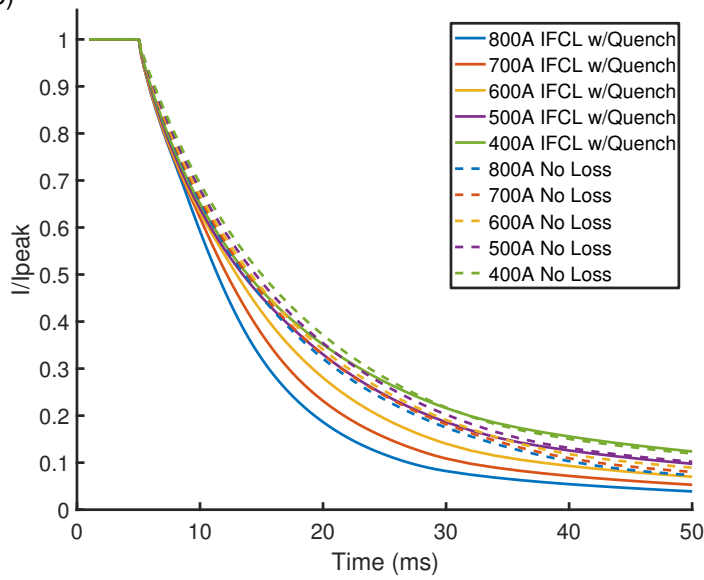

Figure 10. Magnet current decay curves from ANSYS are compared with and without the effects of IFCL and quench for absolute (a) and normalized current (b). The impact of quench back is seen increasing with initial current level. The large thermal margin and smaller rate of field change (driving IFCL) at the $400 \mathrm{~A}$ current level results in the magnet staying superconducting and showing minimal deviation from the no loss case. As the initial current increases up to $800 \mathrm{~A}$, IFCL induced quench plays a larger role, with coil resistance growth driving the current down more quickly than the no loss case.

peak coil hotspot temperature for each initial current level. The quench integral is the time integral of the square of magnet current from the start to end of the decay. This is a material property independent measure of the total energy deposited during the decay which would generate joule heating at a quench location. The quench integral is typically used for an estimation of quench location hotspot temperature in the adiabatic limit with the material properties considered [19], and in this case serves as a metric for the degree of quench back when compared to a no loss case. In the future, a more detailed metric could be used along with an exploration of combined effects due to multi-parameter deviation from the nominal case.

The results show the most sensitive fits and parameters are those associated with the resistivity of the copper matrix ( $f_{\text {eff }}, \mathrm{Cu} \mathrm{RRR}$, and $\mathrm{Cu}$ resistivity fit). This is not unexpected, as these impact the IFCL time constant and coil resistance. A range of behavior about the nominal case due to changes in $\mathrm{Cu}$ resistivity was created to visualize this sensitivity when comparing to test data. This range is bounded by two curves which represent the extremes of the material property fits and RRR. One curve corresponds to a RRR of 150 and the fit from CUDI, and the other a RRR of 350 and the fit from MATPRO. Figure 11 compares this range and the nominal case to test data. Current decay curves are shown from 400 to $800 \mathrm{~A}$, along with the quench integral of these decays from 5 to $50 \mathrm{~ms}$. The ANSYS simulations are seen reproducing the trend seen in the measured data of larger deviation from the no loss case at higher current. The source of the remaining difference between the ANSYS predictions and test data could originate from the 2D elements not including longitudinal quench propagation or 3D effects on peak field, inductance, and coupling loss. In addition, further study of the accuracy 
Table 6. Sensitivity Study of ANSYS Results for the SCU

\begin{tabular}{|c|c|c|c|c|c|c|c|}
\hline \multirow[b]{2}{*}{ Parameter $/$ Fit $^{b}$} & \multirow[b]{2}{*}{ Nominal } & \multirow[b]{2}{*}{ Changed } & \multicolumn{5}{|c|}{ Variation of Results ${ }^{a}: \Delta Q_{i n t}(\%), \Delta T_{h o t}(\mathrm{~K})$} \\
\hline & & & $400 \mathrm{~A}$ & $500 \mathrm{~A}$ & $600 \mathrm{~A}$ & $700 \mathrm{~A}$ & $800 \mathrm{~A}$ \\
\hline \multirow{2}{*}{$f_{\text {eff }}$} & \multirow{2}{*}{4.0} & 2.0 & $-4.6,3.0$ & $-6.4,2.1$ & $-4.7,0.7$ & $-3.0,0.1$ & $-1.1,0.0$ \\
\hline & & 6.0 & $2.3,-1.3$ & $4.5,-5.9$ & $5.6,-1.3$ & $3.8,-0.5$ & $2.4,-0.2$ \\
\hline \multirow{2}{*}{$\mathrm{Cu} \mathrm{RRR}$} & \multirow{2}{*}{250} & 150 & $1.9,-1.0$ & $3.2,-0.8$ & $0.4,1.3$ & $-2.3,1.7$ & $-4.2,1.8$ \\
\hline & & 350 & $-1.1,0.4$ & $-1.2,0.0$ & $0.0,-0.6$ & $1.1,-0.8$ & $2.1,-0.9$ \\
\hline \multirow{2}{*}{$\mathrm{Cu}$ resistivity } & \multirow{2}{*}{ NIST } & CUDI & $0.9,-0.5$ & $1.9,-0.8$ & $0.4,0.7$ & $-1.6,1.4$ & $-3.7,2.2$ \\
\hline & & MATPRO & $-0.1,0.0$ & $0.0,-0.2$ & $0.5,-0.6$ & $1.4,-1.0$ & $2.4,-1.4$ \\
\hline $\mathrm{Cu}$ heat capacity & NIST & CUDI & $0.0,0.0$ & $0.0,0.0$ & $0.0,-0.1$ & $0.0,-0.1$ & $0.1,-0.2$ \\
\hline $\mathrm{Cu}$ thermal cond. & NIST & CUDI & $0.0,0.0$ & $0.0,0.0$ & $0.0,0.0$ & $0.0,0.0$ & $0.0,0.0$ \\
\hline $\mathrm{Nb}_{3} \mathrm{Sn}$ heat capacity & NIST & CUDI & $0.0,-0.4$ & $1.3,-1.0$ & $1.9,0.0$ & $1.8,0.5$ & $1.7,1.4$ \\
\hline G10 heat capacity ${ }^{c}$ & NIST & Fermilab & $0.0,0.6$ & $-2.6,1.5$ & $-3.4,0.8$ & $-3.7,0.4$ & $-3.8,0.0$ \\
\hline G10 $\perp$ therm. cond. & CryoComp & NIST & $0.0,-0.4$ & $0.9,-1.1$ & $0.9,-0.9$ & $0.9,-0.7$ & $1.0,-0.7$ \\
\hline
\end{tabular}

${ }^{a}$ based on deviation of the results from the nominal case for the variation of the single listed parameter. $\Delta Q_{\text {int }}$ is the change in quench integral and $\Delta T_{h o t}$ is the change in peak hotspot temperature of the coil; ${ }^{b}$ all material property fits can be found in [31]; ${ }^{c}$ G10 properties (normal) are used for the epoxy impregnated glass fiber region between strands

of equivalent magnetization models for coupling currents in this application may be revealing. Despite room for further study, the level of agreement between ANSYS and test data is a promising sign the user elements can be used to understand and predict quench back effects in $\mathrm{Nb}_{3} \mathrm{Sn}$ SCU's. 

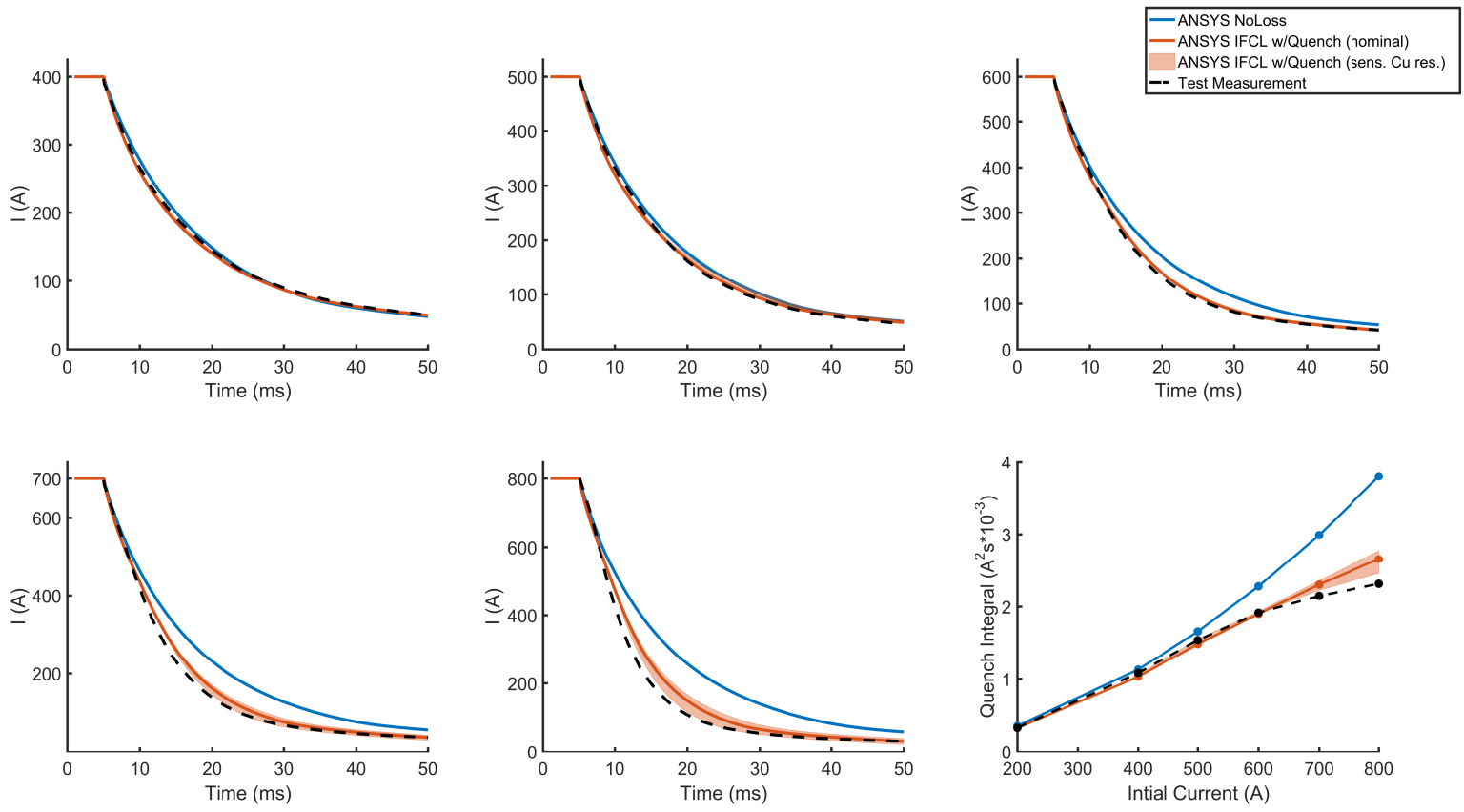

Figure 11. Comparison of ANSYS results with test data shows both exhibiting similar quench back behavior over a range of initial currents. Deviation from the no loss cases is minimal at low current and increases with current level as quench back becomes more prominent. To visualize the sensitivity of the ANSYS model to the parameters and material fits affecting $\mathrm{Cu}$ resistivity, a range is plotted around the nominal case corresponding to the limiting behavior found in the sensitivity study (as described in table 6 and section 5). 


\section{Conclusion}

User defined elements in ANSYS allow for programing the generation of the finite element matrices, providing the opportunity to implement new formulations and material property fits. Two user defined elements were created as a new method for simulating coupled, multiphysics behavior of superconducting magnets. These elements extend the capabilities of ANSYS to now include superconducting specific phenomenon of quench and interfilament coupling loss, while maintaining the meshing, solving, and post-processing capabilities of the standard distribution. These new elements were shown benchmarked against existing codes for a $\mathrm{Nb}_{3} \mathrm{Sn}$ dipole and compared to test data for a $\mathrm{Nb}_{3} \mathrm{Sn}$ prototype undulator. In both cases the user elements were shown predicting IFCL induced quench back, demonstrating for the first time that ANSYS can be used to simulate this strongly coupled behavior required for accurate modeling of many superconducting magnets.

This work is part of a larger effort within the US Magnet Development Program and the Berkeley Center for Magnet Technology to advance analysis and modeling capabilities for superconducting magnets [32]. It is our goal that this work becomes a tool usable by the magnet design community. The effort to make these elements available is underway, and interested parties are encouraged to contact the authors for more information. Future work is focused on the extension of this approach to 3D and towards simulation of HTS coated conductors and bulk superconducting devices by implementing the $E-J$ power law model within the $\boldsymbol{A}-V$ formulation.

\section{Acknowledgments}

Lucas Brouwer would like to thank Emmanuele Ravaioli of CERN (previously LBNL) for many interesting and helpful discussions about the simulation of dynamic effects in superconducting magnets and CLIQ based magnet protection. This work was supported by the Director, Office of Science, High Energy Physics, and U.S. Department of Energy under contract No. DE-AC02-05CH11231 in the context of broader collaboration with the US Magnet Development Program.

\section{Appendix}

The matrices used for the 2D electromagnetic element with circuit coupling found in equations 16 and 17 are the result the following steps. The $K^{A A}$ and $C^{A A}$ matrices in 2D are found by simply reducing the general form already given in Equations 8 and 9 . For the stranded conductor, the source term is now supplied by the circuit and determines the form of $K^{A i}$. Considering the weak integral of the source term in 2D ( $\boldsymbol{J}$ is now a scalar $J_{z}$ ), and that the current density of the stranded conductor is derived from the current per turn $i$ using $J_{z}=\frac{N_{c}}{S_{c}} t i$ leads to 


$$
\int\{N\} J_{z} d S=\int\{N\} \frac{N_{c}}{S_{c}} t\{N\}^{T}\{i\} d S,
$$

where $\{N\}$ is a vector of shape functions carrying the DOF across the element based on the values at the element nodes $\left(i=\{N\}^{T}\left\{i_{e}\right\}\right.$ for example). Here clearly the $K^{A i}$ matrix is given by

$$
\left[K^{A i}\right]=-\frac{t N_{c}}{S_{c}} \int\{N\}\{N\}^{T} d S_{\text {elem }} .
$$

The 2D matrices in the voltage balance are derived from Equations 11, 13, and 15. The $e$ term is re-written as an area integral with the shape functions added, such that

$$
\begin{aligned}
& t \frac{N_{c}}{S_{c}} L_{i} \int\{N\}^{T} \frac{\partial}{\partial t}\{A z\} d S-\frac{1}{S_{c}} \int\{N\}^{T}\{e\} d S \\
& \quad+L_{c}\left(\frac{N_{c}}{S_{c}}\right)^{2} \frac{I_{f c u}}{f_{\text {cond }}\left(1-f_{s c}\right)} \rho_{s t} \int\{N\}^{T}\{i\} d S=0 .
\end{aligned}
$$

ANSYS assembles the three DOF using the following form

$$
\left[\begin{array}{ccc}
C^{A A} & 0 & 0 \\
C^{e A} & 0 & 0 \\
0 & 0 & 0
\end{array}\right]\left[\begin{array}{c}
\frac{\partial A}{\partial t} \\
0 \\
0
\end{array}\right]+\left[\begin{array}{ccc}
K^{A A} & 0 & K^{A i} \\
0 & K^{e e} & K^{e i} \\
0 & 0 & 0
\end{array}\right]\left[\begin{array}{c}
A \\
e \\
i
\end{array}\right]=\left[\begin{array}{l}
0 \\
0 \\
0
\end{array}\right]
$$

To match the sizing of the submatrices here, the vectors in Equation A.3 are expanded to square matrices using the outer product with an identity vector $\{I\}$. This leads to $2 \mathrm{D}$ element matrices of

$$
\begin{gathered}
{\left[C^{e A}\right]=t \frac{N_{c}}{S_{c}} L_{i} \int\{I\}\{N\}^{T} d S_{\text {elem }}} \\
{\left[K^{e e}\right]=-\frac{1}{S_{c}} \int\{I\}\{N\}^{T} d S_{\text {elem }}} \\
{\left[K^{e i}\right]=L_{c}\left(\frac{N_{c}}{S_{c}}\right)^{2} \frac{I_{f c u}}{f_{\text {cond }}\left(1-f_{\text {sc }}\right)} \rho_{\text {st }} \int\{I\}\{N\}^{T} d S_{\text {elem }}}
\end{gathered}
$$

The user element is coupled to an external circuit as voltage source using the standard distribution, circuit element CIRCU124 [22]. With key option one set to select a stranded coil, this element consists of three nodes labeled $i, j$, and $k$. The first two nodes are connected to adjacent circuit elements and each carry a single voltage DOF. The third node carries both a current and voltage drop DOF, and is chosen as one of the nodes in the meshed coil region to make it part of the coupled set. The stiffness matrix for the stranded coil CIRCU124 element is given by 


$$
\left[\begin{array}{cccc}
0 & 0 & 1 & 0 \\
0 & 0 & -1 & 0 \\
-1 & 1 & 0 & s \\
0 & 0 & 0 & 0
\end{array}\right]\left[\begin{array}{c}
V_{i} \\
V_{j} \\
i_{k} \\
e_{k}
\end{array}\right]=\left[\begin{array}{l}
0 \\
0 \\
0 \\
0
\end{array}\right],
$$

where $s$ is a factor to account for modeling of a symmetric region. This couples the stranded coil into an external circuit which may be made up of additional coil regions or generic circuit elements selected using other key options for CIRCU124. 


\section{References}

[1] ROXIE: a Computer Code for the Integrated Design of Accelerator Magnets URL https: //cern.ch/roxie

[2] Guo X L, Wang L and Green M A 2012 Cryogenics 52420

[3] Marinozzi V, Sorbi M, Manfreda G, Bellina F, Bajas H and Chlachidze G 2015 Physical Review Special Topics - Accelerators and Beams 18032401

[4] Breschi M, Cavallucci L, Ribani P L, Gavrilin A V and Weijers H W 2017 IEEE Trans. Appl. Supercond. 274301013

[5] Ravaioli E, Auchmann B, Maciejewski M, ten Kate H H J and Verweij A P 2016 Cryogenics 80 346

[6] Bortot L, Auchmann B, Cortez-Garcia I, Fernandez-Navarro A M, Maciejewski M, Prioli M, Schops S and Verweij A 2018 IEEE Trans. Appl. Supercond. 547000404

[7] Schops S, Gersem H D and Bartel A 2010 IEEE Trans. Magn. 463233

[8] Bortot L, Auchmann B, Cortez-Garcia I, Fernandez-Navarro A M, Maciejewski M, Mentink M, Prioli M, Ravaioli E, Schops S and Verweij A 2018 IEEE Trans. Appl. Supercond. 284900706

[9] ANSYS URL https://www. ansys.com

[10] Caspi S, Chiesa L, Ferracin P, Gourlay S A, Hafalia R, Hinkins R, Lietzke A F and Prestemon S 2003 IEEE Trans. Appl. Supercond. 131714

[11] Yamada R, Marscin E, Lee A and Wake M 2004 IEEE Trans. Appl. Supercond. 14291

[12] Ferradas-Troitino J, Ambrosio G, Bajas H, Bordini B, Ferracin P, Fleiter J, Izquierdo-Bermudez S, Vicente-Lorenzo-Gomez J, Carlos-Perezand J, Vallone G and Senatore C 2019 IEEE Trans. Appl. Supercond. 294701306

[13] Brouwer L, Arbelaez D, Caspi S, Marchevsky M and Prestemon S 2018 IEEE Trans. Appl. Supercond. 284001006

[14] Hauser A 1997 IEEE Trans. Magn. 331572

[15] Testoni P 2003 Implemenation in the ANSYS finite element code of the electric vector potential T$\Omega, \Omega$ formulation and its validation with the magnetic vector potential $A-V, A$ formulation Ph.D. thesis University of Cagliari, Italy

[16] COMSOL Multiphysics URL https://www.comsol.com

[17] 2016 ANSYS Mechanical APDL Theory Reference, Release 17.1

[18] Biro O and Preis K 1989 IEEE Trans. Magn. 253145

[19] Wilson M 1983 Superconducting Magnets (Oxford University Press) ISBN 0198548052

[20] Morgan G 1970 Journal of Applied Physics 413673

[21] Louzguiti L, Zani L, Ciazynski D, Turck B and Topin F 2016 IEEE Trans. Appl. Supercond. 26 4700905

[22] Wang J S 1996 IEEE Trans. Magn. 321071

[23] Russenschuck S 2010 Field Computation for Accelerator Magnets: Analytical and Numerical Methods for Electromagnetic Design and Optimization (Wiley-VCH) ISBN 3527407693

[24] Stekly Z J J and Zar J L 1965 IEEE Transactions on Nuclear Science 12367

[25] STEAM: Simulation of Transient Effects in Accelerator Magnets URL https://espace.cern. $\mathrm{ch} / \mathrm{steam} /$

[26] Brouwer L, Auchmann B, Bortot L and Stubberud E 2019 LBNL Eng. Note: SU-1010-4841, R1.0 URL https://usmdp.lbl.gov/scpack-code/

[27] Ravaioli E 2015 CLIQ. A new quench protection technology for superconducting magnets Ph.D. thesis University of Twente, Netherlands

[28] Emma P, Holtkamp N, Nuhn H, Arbelaez D, Corlett J, Myers S, Prestemon S, Schlueter R, Doose C, Fuerst J, Hasse Q, Ivanyushenkov Y, Kasa M, Pile G, Trakhtenberg E and Gluskin E 2014 A plan for the development of superconducting undulator prototypes for LCLS-II and future FELs FEL 2014 Conference Proceedings, Basel, Switzerland p THA03

[29] Zhou C, van Lanen E, Veldhuis D, ten Kate H, Dhalle M and Nihuis A 2011 IEEE Trans. Appl. 
Supercond. 21 2501-2504

[30] Zhou C, Miyoshi Y, van Lanen E, Dhalle M and Nihuis A 2012 Supercond. Sci. and Technol. 25 065018

[31] Manfreda G 2011 CERN Internal Note: EDMS NR 1178007

[32] Gourlay S, Prestemon S, Zlobin A, Cooley L and Larbalestier D 2016 The U.S. Magnet Development Program Plan URL https://www.osti.gov/scitech/servlets/purl/1306334 